\section{Response to: 'Painful knee but not hand osteoarthritis is an independent predictor of mortality over 23 years follow-up of a population-based cohort of middle-aged women' by Gao et al}

We thank Gao $e a^{1}$ for their interest in our paper. ${ }^{2}$

Gao et al highlighted a number of issues related to discordance between pain and radiographic changes and sensitivity of the pain assessment. Although a single definition of knee osteoarthritis (KOA) remains elusive, the presence of knee pain is the main symptom of $\mathrm{KOA}^{3-5}$ and there is still a strong relationship between pain and radiographic knee osteoarthritis (ROA). ${ }^{6} 7$ Notwithstanding this, a substantial number of patients with $\mathrm{ROA}$ are asymptomatic. Although $\mathrm{OA}$ is the most common 'source' of knee pain in ROA, it is true, as pointed out by Gao et al that many other diagnoses may be judged to account for a patient's symptoms including bursitis, tendinopathies and overuse injuries. In this cohort of middle aged to elderly women, we feel that these alternative causes will not be prevalent. We defined pain as the presence of side-specific knee pain in the preceding month, and painful radiographic KOA as kneespecific pain and presence of ROA. We have performed additional analyses to further address the queries of Gao et al.

We analysed symptomatic ROA (SROA) using a definition of pain as present for at least 15 days in the last month, which is commonly used in the literature. ${ }^{89}$ Of the original painful ROA group, $49 \%$ fulfilled the new pain criteria, and a further $21 \%$ reported knee pain on 6-14 days in the preceding month. Both the Kaplan-Meier survival plot (figure 1) and HR for all-cause mortality in the model adjusted for age (2.06 (95\% CI 1.14 to 3.72)) for SROA were very similar to the one presented in our paper for painful ROA group. In the fully adjusted model, a similar trend was seen with a HR of 1.6 (95\% CI 0.77 to 3.44), but it was not statistically significant, most likely due to a small number women in the SROA group $(n=28)$. Furthermore, we excluded from analyses any participants with joint pain due to other diseases that are or might be associated with a decrease in average life expectancy including inflammatory arthritis $(n=43)$. We are confident that the association with mortality in our paper is in women with painful radiographic KOA.

Gao et al mentioned a number of interesting points related to the consequences of KOA or its treatment. Our question related to whether women with KOA suffered or had an increased risk of premature mortality. As such, we adjusted for known potential confounders, but we felt that the consequences and treatment of KOA would be on the causal pathway of the association and that by adjusting for them we would induce a bias towards the null hypothesis. Hence, we did not include them in our model. We agree that it is likely that individuals with KOA are more sedentary and have poor cardiovascular fitness. We therefore performed a sensitivity analysis looking at crude measures of physical activity and found that it did not attenuate the findings, but we accept that the reliability and validity of a single question are limited. We also found no substantial differences in the results when women who underwent knee replacement (from any of the knee subgroups) were excluded during the follow-up.

Although as noted by Gao et al, increased mortality of the painful ROA group compared with the control group could be potentially explained by the higher mean age and body mass index (BMI). Age and BMI were adjusted for in our multivariable models. Furthermore, the painless ROA group (pain-/ROA + ), with similar characteristics, in the fully adjusted model showed no positive association with excess mortality.

Similar to previous studies, we found that knee subgroups with ROA (pain + and pain-) were more likely to have radiographic hand OA as well. ${ }^{10}$ We decided not to include radiographic hand $\mathrm{OA}$ in the knee analyses as it has not been an independent risk factor associated with any excess mortality in this or previous study using Cox proportional hazards models adjusted for known cardiovascular risk factors. ${ }^{11}$ The ability of our statistical model to explain significant mortality differences in hand $\mathrm{OA}$ analyses gives us confidence that our models were adjusted for all-important covariates. This included baseline non-steroidal anti-inflammatory drug use, occupation, age, BMI, typical cardiovascular risk factors, past physical activity and existing cardiovascular disease (CVD). Despite the understandable desire to include as many potential

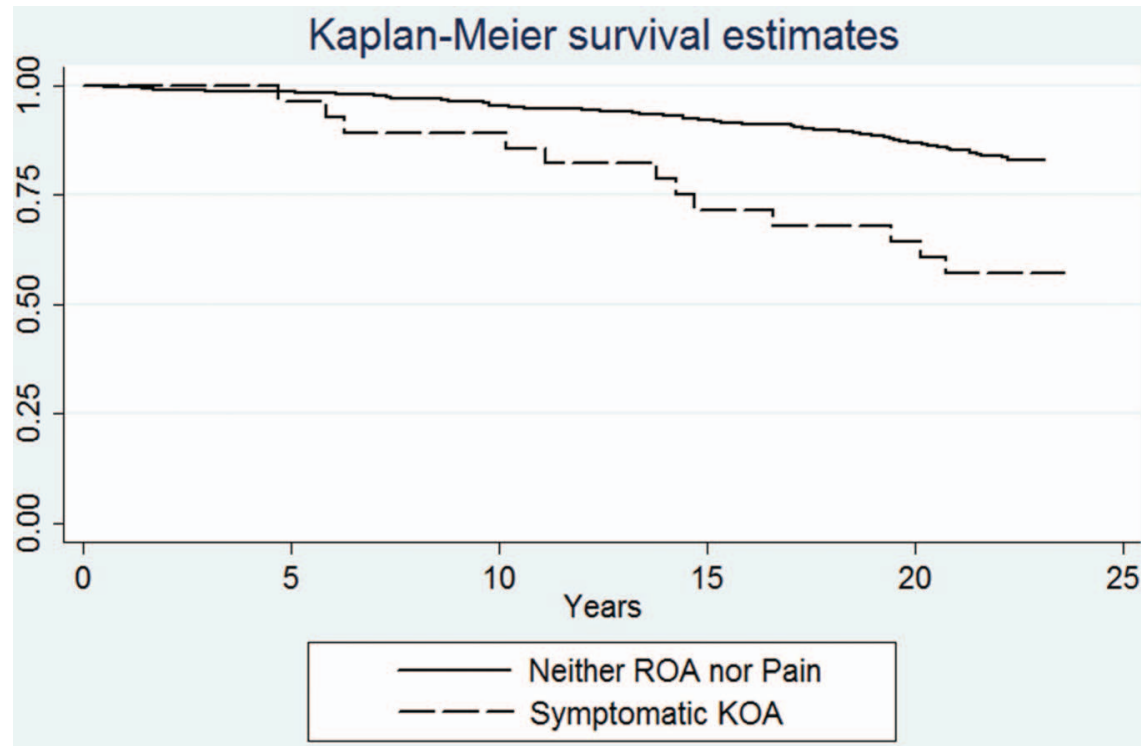

Individuals with ROA and pain on most days in the preceding month were included in the symptomatic KOA group only 
variables as possible in regression analysis, overfitting of the model can be a real problem.

Prospective changes of the risk factors in each subgroup, differences in inflammatory markers, adipokines and health status including future risk of falls and fractures are very interesting new research questions emerging from our findings, and we are planning to investigate them in the future. We agree that it is plausible that painful KOA is an early sign of metabolic dysregulation, leading to CVD problems ${ }^{12}$ and this will be a focus of future research.

\section{S Kluzek, ${ }^{1,2}$ M T Sanchez-Santos, ${ }^{1,2}$ K M Leyland, ${ }^{1,2}$ A Judge, ${ }^{1,3}$ J Newton, ${ }^{1,2}$ N K Arden ${ }^{1,2,3}$}

${ }^{1}$ Oxford NIHR Musculoskeletal Biomedical Research Unit, Nuffield Department of Orthopaedics, Rheumatology and Musculoskeletal Sciences, University of Oxford, Oxford, UK

${ }^{2}$ Arthritis Research UK Centre for Sport, Exercise and Osteoarthritis, University of Oxford, Oxford, UK

${ }^{3}$ MRC Lifecourse Epidemiology Unit, University of Southampton, Southampton General Hospital, Southampton, UK

Correspondence to Dr Stefan Kluzek, Arthritis Research UK Centre for Sport, Exercise and Osteoarthritis, University of Oxford, Old Road, Oxford OX3 7LD, UK; stefankluzek@doctors.net.uk

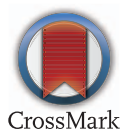

To cite Kluzek S, Sanchez-Santos MT, Leyland KM, et al. Ann Rheum Dis 2016;75: e23.

Received 15 January 2016

Accepted 16 January 2016

Published Online First 10 February 2016

\section{SLinked}

http://dx.doi.org/10.1136/annrheumdis-2015-209026

Ann Rheum Dis 2016;75:e23. doi:10.1136/annrheumdis-2015-209029

\section{REFERENCES}

1 Gao S-G, Zeng C, Xiong Y-lin, et al. Is painful knee an independent predictor of mortality in middle-aged women? Ann Rheum Dis 2016;75:e22.

2 Kluzek S, Sanchez-Santos MT, Leyland KM, et al. Painful knee but not hand osteoarthritis is an independent predictor of mortality over 23 years follow-up of a population-based cohort of middle-aged women. Ann Rheum Dis 2015. Published Online First 5 Nov 2015. doi:10.1136/annrheumdis-2015-208056

3 Finan $\mathrm{PH}$, Buenaver LF, Bounds SC, et al. Discordance between pain and radiographic severity in knee osteoarthritis: findings from quantitative sensory testing of central sensitization. Arthritis Rheum 2013;65:363-72.

4 Hannan MT, Felson DT, Pincus T. Analysis of the discordance between radiographic changes and knee pain in osteoarthritis of the knee. J Rheumato/ 2000;27: 1513-17.

5 Bedson J, Croft PR. The discordance between clinical and radiographic knee osteoarthritis: a systematic search and summary of the literature. $B M C$ Musculoskelet Disord 2008;9:116.

6 Soni A, Kiran A, Hart DJ, et al. Prevalence of reported knee pain over twelve years in a community-based cohort. Arthritis Rheum 2012;64:1145-52.

7 Muraki S, Oka H, Akune T, et al. Prevalence of radiographic knee osteoarthritis and its association with knee pain in the elderly of Japanese population-based cohorts: the ROAD study. Osteoarthritis Cartilage 2009;17:1137-43.

8 Murphy LB, Moss S, Do BT, et al. Annual Incidence of Knee Symptoms and Four Knee Osteoarthritis Outcomes in the Johnston County Osteoarthritis Project. Arthritis Care Res (Hoboken) 2016;68:55-65.

9 Cicuttini FM, Baker J, Hart DJ, et al. Association of pain with radiological changes in different compartments and views of the knee joint. Osteoarthritis Cartilage 1996:4:143-7.

10 Hirsch R, Lethbridge-Cejku M, Scott WW Jr, et al. Association of hand and knee osteoarthritis: evidence for a polyarticular disease subset. Ann Rheum Dis 1996;55: 25-9.

11 Haugen IK, Ramachandran VS, Misra D, et al. Hand osteoarthritis in relation to mortality and incidence of cardiovascular disease: data from the Framingham Heart Study. Ann Rheum Dis 2015;74:74-81.

12 Kluzek S, Newton JL, Arden NK. Is osteoarthritis a metabolic disorder? Br Med Bull 2015;115:111-21. 